\title{
Epidemiology
}

\section{Acute hepatitis B in patients in Britain related to previous operations and dental treatment}

\author{
SHEILA POLAKOFF
}

\begin{abstract}
The frequency of transmission of hepatitis B virus infection from health service staff to patients was assessed from reports of confirmed cases of acute clinical hepatitis in 1980-3. During the four years 4505 reports ( $91 \%$ of the total) included replies to a question about recent operations; 153 patients $(3.4 \%)$ had this history. Transfused blood or blood products were considered the source for 27 cases $(0.06 \%)$. Eleven patients $(0.02 \%)$ were infected in two clusters, both in cardiac surgery units; six were caused by a perfusion technician, who was a symptomless carrier, and five by a surgical registrar during the incubation period of an acute hepatitis $B$ infection. The estimated average annual risk of a patient developing acute hepatitis $B$ as part of a cluster caused by staff during surgical procedures was one in a million operations. For another 11 patients blood transfusion could not be excluded as a source. Where no association between surgery and hepatitis was found the incidence of a history, lay between 2.3 and $2.6 \%$. The Hospital In-Patient Enquiry data showed that about $2.4 \%$ of the population had had operations in a six month period.
\end{abstract}

These findings suggest that transmission of hepatitis B infection from staff to patients is rare in Britain and that the small risk could be eliminated by attention to measures to preserve asepsis and by immunising staff at risk.

\section{Introduction}

The acquisition of hepatitis B infection by health service staff from patients is an occupational hazard, the size of which can be estimated by comparing infection rates in health service staff with those in the general population. ${ }^{12}$ Reports of transmission in the other direction-that is, from staff to patients-are uncommon, and attempts to assess their frequency were considered unnecessary in Britain, where both the incidence of acute hepatitis B and the prevalence of hepatitis $B$ surface antigen $(\mathrm{HBsAg})$ carriage are low. After an outbreak of acute hepatitis B among patients, however, which was caused by a gynaecologist who was a symptomless carrier of the infection, ${ }^{3}$ a study was designed to estimate the frequency of such events. A similar episode, which was discovered in England and investigated locally before plans for the national study were completed, emphasised the need for the investigation (Anonymous Control of Infection Team, unpublished observations).

We decided to make a nationwide study based on confirmed cases of acute hepatitis B which are reported from laboratories through-

Hepatitis Epidemiology Unit, Central Public Health Laboratory, London NW9

SHEILA POLAKOFF, MFCM, MD, consultant epidemiologist out England and Wales-Public Health Laboratory Service (PHLS) communicable disease reports. The objects of the study were to find out how often clusters of cases of acute hepatitis B occur after surgery or dental treatment in Britain and also to establish how often surgical procedures were recorded as being carried out in the six months before the onset of acute hepatitis B and to compare this rate with the corresponding rate in the general population.

\section{Methods}

The special records used for hepatitis B reports to the PHLS were adapted to include an explicit inquiry about surgery or dental procedures in the six months before the onset of the illness. Directors of all microbiological laboratories reporting hepatitis $B$ infections were asked to have the records completed and also, for each patient who had had an operation or dental treatment in the relevant period, to obtain clinical information and the following details for entry in a special study record: the name of the hospital or establishment where the operation was performed, the date and type of operation, and the product number of any blood or blood product transfused. For dental treatment in general practice the postal district of the dental surgery in which the patient had been treated was required. The names of the surgeons, dentists, or any other health service staff who took part in these procedures were not recorded.

All records were sent to the Hepatitis Epidemiology Unit, Central Public Health Laboratory, where they were inspected for evidence of clustering, in time or place, of procedures undergone by patients before the onset of hepatitis.

A cluster possibly associated with surgical or dental treatment was defined as follows: two patients who, within 12 months, had undergone (a) operations in the same hospital, hospital group, or other establishment: or (b) dental treatment at any surgery in any one postal district. When a possible cluster was identified, more detailed inquiries were made to find out if there were any common features in the procedures-for example, types of operation, their time and place, and staff taking part. If a relation was found further investigations similar to those described previously were made: these included tests for hepatitis B surface antigen ( $\mathrm{HBsAg}$ ) of blood from staff concerned in the procedures, HBsAg subtyping, and follow up studies of groups of patients who had operations in which the staff carrier was or was not involved. ${ }^{3}$

\section{DIAGNOSIS OF ACUTE HEPATITIS B}

Patients with illnesses diagnosed by their physicians as acute clinical hepatitis and whose serum contained $\mathrm{HBsAg}$ were included in the study. Cases of acute clinical hepatitis without detectable $\mathrm{HBsAg}$ were included if antibody to hepatitis B core antigen (anti-HBc) was found and other circumstances-for example, first serum not taken until the late or convalescent stage of the illness or identification of the immunoglobulin fraction $\mathrm{M}$ of anti-HBc (anti-HBc IgM) - suggested its importance.

\section{SERUM SAMPLES AND LABORATORY TESTS}

Serum samples from patients were tested for HBsAg at local laboratories by one of the following methods: immunoelectrophoresis, passive haemag- 
glutination, radioimmunoassay, or enzyme linked immunosorbent assay. In some cases two or three of these methods were used.

Anti-HBc tests were made by radioimmunoassay at the Virus Reference Laboratory at the Central Public Health Laboratory.

A portion of serum from each patient with a history of any surgical or dental procedure in the relevant period was sent for storage at the Virus Reference Laboratory.

Serum samples from individuals in possible clusters were examined for HBsAg subtypes by radioimmunoassay at the Department of Virology, Middlesex Hospital Medical School, London.

\section{BLOOD TRANSFUSION IN SIX MONTHS BEFORE ONSET OF ILLNESS}

Although all blood for transfusion was screened at source for HBsAg by sensitive methods during the study period, the possibility that blood or its products might be the source of the infection was investigated at the relevant regional blood transfusion centre by examining stored samples, if available, and by recalling donors for further tests.

Hepatitis was considered certainly, probably, or possibly due to blood transfusion as follows

Certain - (a) tests of stored samples of donated blood contained HBsAg or anti-HBc IgM or (b) follow up donor serum showed evidence of hepatitis $B$ virus infection since the donation, with or without clinical hepatitis.

Probable-(a) Only anti-HBc was identified in follow up donor serum or (b) multiple transfusions $(>70)$ - that is, too many to allow follow up.

Possible-Anti-HBc and anti-HBs were found in follow up donor serum sample.

\section{ESTIMATE OF INCIDENCE OF SURGICAL PROCEDURES IN GENERAL POPULATION}

The Hospital In-Patient Enquiry routinely takes a 10\% sample of records of patients in National Health Service hospitals in England and Wales to obtain information on many details related to patient care, including discharges (and deaths) from hospitals after operations. ${ }^{4}$ These records provide a guide to the annual incidence of surgical procedures in the general population, although as some patients have more than one procedure in a year the rates are slightly higher than rates for patients. All assisted births were included in the inquiry until 1981.

\section{Results and comment}

During 1980-3 4934 reports of patients with acute hepatitis B were received, $4804(97 \%)$ of whom had detectable $\mathrm{HBsAg}$ at the time of sampling. Of these reports $4505(91 \%)$ included replies to a question about operations and dental treatment in the previous six months. The reply rate did not vary much between areas or between patients in age and sex groups, but it was notably lower for patients whose hepatitis attacks were diagnosed at clinics for sexually transmitted diseases.

Of the 169 patients who had had operations; 16 had had their operations abroad. Dental treatment was recorded for 268 patients.

\section{POSSIBLE CLUSTERS}

Nineteen instances met the criteria for a possible cluster: eight were related to operations and 11 dental treatment (table I).

TABLE I-Possible clusters associated with surgery and dentistry

\begin{tabular}{|c|c|c|}
\hline $\begin{array}{l}\text { Results of } \\
\text { investigation }\end{array}$ & $\begin{array}{l}\text { No of } \\
\text { clusters }\end{array}$ & Details \\
\hline \multicolumn{3}{|l|}{ Surgery ${ }^{\star}$} \\
\hline Cases not related & 6 & $\begin{array}{l}2 \text { patients in each of } 5 \text { clusters } \\
3 \text { patients in } 1 \text { cluster }\end{array}$ \\
\hline $\begin{array}{l}\text { Cluster } \\
\text { confirmed }\end{array}$ & 2 & $\begin{array}{l}6 \text { patients in cardiac surgery unit } \\
\text { Source: perfusion technician, who was a } \\
\text { symptomless carrier } \\
5 \text { patients in cardiac surgery unit } \\
\text { Source: registrar in incubation period }\end{array}$ \\
\hline \multicolumn{3}{|l|}{ Dental treatment } \\
\hline Cases not related & 10 & $\begin{array}{l}2 \text { patients in each of } 7 \text { clusters } \\
3 \text { patients in } 3 \text { clusters }\end{array}$ \\
\hline $\begin{array}{l}\text { Information not } \\
\text { available }\end{array}$ & 1 & 2 patients \\
\hline
\end{tabular}

*One patient developed acute hepatitis $B$ after an operation in which a house officer in the incubation period of an attack of hepatitis $B$ participated.
TABLE II-Hospital In-Patient Enquiry: $10 \%$ sample of all dischanges after all operations and procedures

\begin{tabular}{lrrrrr}
\hline Age (years) & $<15$ & $15-44$ & $45-64$ & $\geqslant 65$ & Total \\
Men & 5166300 & 10507900 & 5468800 & 2977700 & 24120700 \\
$\quad$ Population & 19043 & 29462 & 24843 & 24620 & 107968 \\
$\quad$ No of patients discharged & $3 \cdot 69(1 \cdot 8)$ & $2 \cdot 80(1 \cdot 4)$ & $4 \cdot 54(2 \cdot 3)$ & $8 \cdot 27(4 \cdot 1)$ & $4 \cdot 48(2 \cdot 2)$ \\
$\quad$ Rate percent & & & & & \\
Women & 4902300 & 10270500 & 5705000 & 4594400 & 25472200 \\
$\quad$ Population & 12369 & 60049 & 28552 & 26594 & 127564 \\
$\quad \begin{array}{l}\text { No of patients discharged } \\
\text { Rate percent }\end{array}$ & $2 \cdot 52(1 \cdot 3)$ & $5 \cdot 85(2 \cdot 9)$ & $5 \cdot 00(2 \cdot 5)$ & $5 \cdot 79(2 \cdot 9)$ & $5 \cdot 01(2 \cdot 5)$
\end{tabular}

^Procedures include all assisted births; discharges include deaths.

†Rate percent $=\frac{\text { No of discharges } \times 10}{\text { Total population }} \times 100$. Estimated rate percent per six months in parentheses.

Hospital In-Patient Enquiry 1981.

Surgery

There were two or three patients in each of six possible clusters, none of which showed any association in time, place, or surgical team.

Infections were clearly associated with operations in two clusters, both affecting patients who had had cardiac surgery. In the first of these two clusters six patients developed acute hepatitis within six months of their operations in one unit. This unit employed a perfusion technician who had clinically recovered from a mild anicteric illness, during which he was found to be $\mathrm{HBsAg}$ positive, subtype ay, with the serological marker of high infectivity, hepatitis $\mathrm{Be}$ antigen ( $\mathrm{HBeAg})$. In accordance with recommenda- iv tions ${ }^{5}$ he was allowed to return after expert counselling on specific measures to avoid transmission of infection, including the wearing of gloves at all times during work, since at that time no cases of hepatitis could have been attributed to him. Nevertheless, acute hepatitis B, subtype ay, developed in six of the patients who had had operations in the unit during an 18 month period after the carrier had returned to work there. There was no common feature other than the carrier perfusionist. The donors whose blood was $c$ given to the first two patients were still being traced when the third case was reported. The carrier was then transferred to other duties while detailed studies were made. These showed that there were no more than the six patients with clinical hepatitis and that none of the patients who had had operations in the unit in the 18 months after the carrier has been excluded developed the illness. Despite counselling it appeared that the carrier had failed to wear gloves at work and had often had cuts and abrasions of the skin of his hands. He accepted a training course for non-clinical work.

In another cardiac surgery unit acute hepatitis $B$ developed in five patients after their operations. In each case a registrar had taken part in the operation before the obvious onset of hepatitis, though he had been feeling increasingly unwell for several weeks. The five operations took place over only 18 days. Follow up studies of patients who had surgical treatment by the team which had included the registrar in the two months after he took sick leave showed none with clinical hepatitis. All six infections were HBsAg, subtype ad. The registrar became $\mathrm{HBsAg}$ negative within weeks of the onset of illness.

No other infections which met the cluster criteria were found, but one patient developed acute hepatitis after an operation in which a house officer had taken part during the incubation period of an attack of acute hepatitis. Both infections were $\mathrm{HBsAg}$ positive, subtype ad.

\section{Dental treatment}

Eleven possible clusters concerned patients who had had dental treatment in the six months before the onset of acute hepatitis B. Two patients were identified in each of eight clusters and three patients in each of three clusters. In 10 instances it was possible to identify the surgeries at which the patients had been treated. In all but three possible clusters the patients had been treated at different surgeries by different dentists and any association between the infections in the other three was excluded by further investigation. In one instance the two patients had been treated at the same dental hospital; although the operators were not identified the subtype of one infection was ad and the other ay. In the two other possible clusters each of the pairs of patients had been treated at different times by the same dentist. Serum samples from the two dentists and a nurse who had helped in the treatment of one pair of patients showed no evidence of hepatitis B virus infection.

\section{BLOOD TRANSFUSION}

As well as detecting clusters the study was designed to estimate the incidence of recent surgery among patients with acute hepatitis $B$ and, if this (1) 
differed from the rate among the general population, to try to assess how much might be attributable to infections from staff. Post-transfusion hepatitis $\mathrm{B}$, which would increase the incidence, needed to be determined and excluded.

Of the 153 patients, 56 had received blood (or plasma) transfusions and two haemophiliacs had been given large amounts of cryoprecipitate at the time of operation. Blood transfusion was considered the certain source of infection in 14 cases $(24 \%)$ and the probable source in 13 others $(22 \%)$ including those who had received cryoprecipitate. In three cases the serological evidence was not clear and in another eight the follow up of donors was incomplete (19\%). Blood was excluded as a source in 20 cases $(34 \%)$. None of the cases in the possible clusters was attributable to transfusion.

PREVIOUS OPERATIONS IN GENERAL POPULATION AND IN PATIENTS WITH ACUTE HEPATITIS B

Statistics from the Hospital In-patient Enquiry for the year 1981 are the latest available. They may provide an under estimate for the years 1980-3 inclusive since there was a slight but sustained upwards trend in the rates of discharge from hospital during 1979-81.

The inquiry showed that for men the annual rate of operations was $4.5 \%$; the corresponding rate for women was $5 \cdot 0 \%$. Annual rates in age groups ranged from $2 \cdot 5 \%$ in girls under the age of 15 to $8.3 \%$ in men of 65 and more (table II). On this evidence, slightly less than $2.4 \%$ of the general population interviewed at any time should have a history of some type of surgical procedure, as defined, in the previous six months and the expected rate should vary from $1 \cdot 3$ to $4 \cdot 1 \%$ according to age and sex.

Of the 4505 patients with reports that included a reply to the question about surgery in the previous six months, $153(3.4 \%)$ had this history (table III). Of these, $27(0 \cdot 6 \%)$ were ascribed to blood transfusion and $11(0 \cdot 2 \%)$ were identified as part of clusters caused by staff. A further $11(0 \cdot 2 \%)$ were possibly caused by blood transfusion. Thus, causes related to operations were not found for $2 \cdot 3$ to $2 \cdot 6 \%$ of the patients. This is similar to the estimated $2 \cdot 4 \%$ of discharges after operations based on Hospital Inpatient Enquiry data.

TABLE III-Summary of data on operations in patients with acute hepatitis $B$

\begin{tabular}{lc}
\hline & Number (\%) \\
\hline Reports of acute hepatitis B in patients & 4934 \\
Reports with reply to question of previous surgery or dentistry & 4505 \\
Patients who had had operations in Britain & $153(3 \cdot 4)$ \\
Patients with definite or probable source of infection at operation & $27(0 \cdot 6)$ \\
$\quad$ Blood transfusion (includes 2 given cryoprecipitate) & $11(0 \cdot 2)$ \\
Staff associated clusters & $11(0 \cdot 2)$ \\
Patients with blood transfusion as a possible surce & $115(2 \cdot 6)$ \\
Patients without definite or probable source & $104(2 \cdot 3)$ \\
Patients without definite, probable, or possible source & $2 \cdot 4 \%$ \\
Crude rate of discharge after operation from hospital in six months & $268(5 \cdot 9)$ \\
Patients with a history of dentistry & 30493000 \\
Annual number of courses of dental treatment in England and Wales $\dagger$ &
\end{tabular}

^Includes one patient not included in cluster probably infected by staff at operation.

†1981 figures. DHSS Governmental Statistical Service. Health and Personal Social Services, p 77. Welsh Office Governmental Statistical Service. Health and Personal Social Services, p 50 .

A more detailed comparison of the study results and the Hospital Inpatient Enquiry data was based on the numbers obtained in the study and the numbers expected in age and sex groups (table IV). The study rates are overestimates, because 32 patients for whom sex was not stated and 222 for whom age was not stated, among whom only one had had an operation, were excluded. Among men, the figure of 64 patients who had had operations, including nine who had possibly acquired their infection from blood transfusion, was greater than the expected 48 , which was at the lower limit of the approximate $95 \%$ confidence interval when the nine were included, but within the interval when they were excluded. Among women, 51 patients had had operations, including two who had possibly acquired the infection from blood transfusion, whereas the expected number was 39 . Both estimates for women lay within the approximate $95 \%$ confidence intervals.

Whether these differences were real or chance variations, they cannot necessarily be ascribed only to infections associated with previous operations. There are other variables which would add to any difference due to previous operations. One of these is any lifestyle which increases the risk of both hepatitis $B$ infection and conditions which require surgery. Another is any selection, on the basis of the history of recent operations, of patients with hepatitis for whom serological confirmation of diagnosis is requested. The amount contributed by each variable separately could not be determined.
TABLE IV-History of surgery in patients with acute hepatitis $B$ and rates of discharge from hospital after surgical procedures in six month period

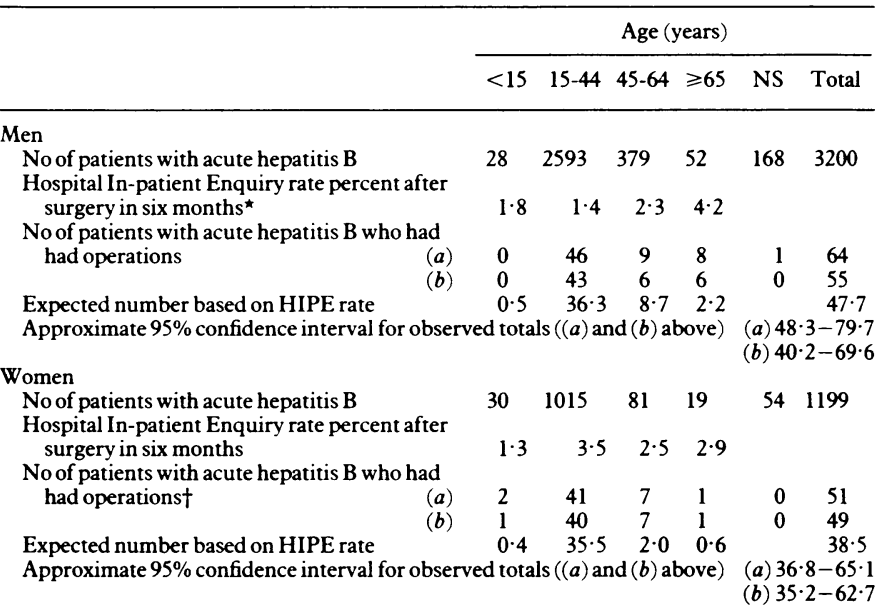

(a) Excludes patients who had operations abroad and infections acquired in clusters or from blood transfusion.

(b) As $(a)$ but also excluding infections possibly acquired from blood transfusion

*Based on Hospital In-patient Enquiry data for 1981 (last year in which data for England and Wales were combined). Number of discharges and deaths after surgical procedures in six months period in age and sex groups in population. tIncludes assisted deliveries.

\section{RATES OF PREVIOUS DENTAL TREATMENT}

Of the 4505 patients, $268(5.9 \%)$ had received dental treatment in the six months before the onset of hepatitis. The only routinely collected data for comparison are the number of NHS courses of dental treatment, which were more than 30 million in 1981 for a population of approximately 50 million (table III). If these courses were distributed evenly throughout the population $30 \%$ of individuals should be expected to have had dental treatment in the six months before the onset of illness.

\section{INCIDENCE OF ACUTE CLINICAL HEPATITIS B IN SURGEONS AND DENTISTS}

During the period of the study 12 surgeons and eight dentists with acute hepatitis B were reported, an average annual rate of 25 per 100000 and 13 per 100000 respectively.

\section{Discussion}

The reliability of this type of study depends on the comprehensiveness of the reporting system. Several factors contribute to a high level of reporting of acute hepatitis B in the PHLS reports: awareness of doctors of the possibility that hepatitis, particularly in adults, may be due to type $B$ infection; the ready availability of laboratory facilities for hepatitis $B$ virus marker tests throughout the country; and the willingness of almost all laboratories undertaking these tests to report to the PHLS. Arrangements made between biochemical and microbiological laboratories in some areas for a portion of any serum specimen with a possible diagnosis of hepatitis submitted for biochemical tests to be sent on for HBsAg tests contribute further to identifications. A comparison of the PHLS reports of acute hepatitis $B$ with the results of a detailed study of the incidence of hepatitis in a population of two thirds of a million in an area of London indicated that the reports provided as good an estimate of the incidence of acute hepatitis $B$ as the population study $^{6}$ and that the reports should represent most cases of acute hepatitis B in the country as a whole.

The first aim of the study was to find out whether clusters of hepatitis B infections caused by operators during surgical or dental procedures were as rare in Britain as had been supposed. Only two clusters were found in the four years: neither was caused by a persistent carrier surgeon or dentist, though both affected patients having operations. The seven infections caused by a carrier perfusionist in a cardiac unit could probably have been avoided if the advice given about the wearing of gloves had been heeded. It was 
not possible to identify the route of entry of infection to the patients' circulation, but for this to have happened there must have been some breakdown in the application of principles of asepsis. The six infections caused by a registrar took place during the short period in which he continued to work while incubating hepatitis, though jaundice was not apparent. This surgeon's infection, and the subsequent events, could have been prevented by previous active immunisation or, failing that, the patients' infections might have been avoided if he had not persisted in working while he was unwell.

There were no clusters associated with dental treatment. Twice in the four years two patients formed a possible cluster associated with dental treatment, as defined in the study, but investigations showed that the infections could not have been acquired from the dentists, who had no serological evidence of hepatitis B virus infection, and the patients' treatments were not related in time.

Apart from the detection of clusters, the aim of this study to assess the size of the risk that infection transmitted sporadically by health service staff during surgical or dental treatment might pose by comparing the incidence of recent operations in patients and in the general population. The Hospital In-Patient Enquiry afforded data for the comparison and, moreover, showed clearly that operations are so common in the general population that a portion of patients with acute hepatitis B must be expected to have this history by chance. Among the study patients the proportion who had had operations was of the same order as that shown by the Hospital Inpatient Enquiry, but the proportion among the study patients was higher after standardisation for age and sex. This difference could not, however, be necessarily attributed solely to infections acquired during operations since other factors, such as particular lifestyles or patient selection, would contribute to it. For example, sexual promiscuity, which increases the risk of acquiring hepatitis $\mathrm{B}$, also tends to lead to gynaecological conditions which require operations. Also, for both sexes, sexual promiscuity and the abuse of alcohol or drugs increases the risk of hepatitis $B$ and also the chance of road traffic accidents, the results of which often require surgical treatment.

Although the effects of these variables could not be disassociated the comparison made it possible to put the risk of acquiring hepatitis $B$ during an operation into perspective. The Hospital In-patient Enquiry data show that more than two and a quarter million operations are performed annually in England and Wales. The study results show that the risk of acquiring acute hepatitis B as part of a cluster associated with surgery is about one in a million. If we assume that all of the difference between the number of patients with hepatitis $B$ with a history of recent operations and the number expected on the basis of Hospital In-patient Enquiry data was due to sporadic infections acquired during the operation (and this is improbable) their estimated number in addition to those acquired in the cluster would increase the risk to no more than one in 250000 . In comparison, the risk to staff from patients, though not large, is much greater. For example, during the period of the survey the average annual rate of acute hepatitis $B$ among surgeons was 25 per 100000 , a value at least a hundred times greater than that estimated for patients.

The finding of low rates of transmission does not, however, diminish the need to reduce them. This could be achieved by strict attention by all staff taking part in operations to measures to preserve asepsis, adaption of techniques to reduce the chance of sharps injuries, and, now that safe and effective vaccines are available, immunisation of theatre staff working in units in which the risk of acquiring the infection from patients is high.

The absence of clusters associated with dental treatment and the low incidence of recent dental treatment among patients with acute hepatitis B in terms of the annual number of courses of NHS dental treatment in England and Wales did not suggest an association between dental treatment and hepatitis $B$.

This study was made throughout England and Wales in collaboration with consultants in charge of more than 80 microbiological laboratories, where hepatitis B virus marker tests are undertaken, directors of all Blood Transfusion Centres, as well as directors of Public Health Laboratories.

Statistical analyses were made by Dr Hilary Tillett and Mrs Janet Mortimer and computer analyses by Dr G Manning and Mrs Margaret Pipe, PHLS Communicable Disease Surveillance Centre.

The study was designed and coordinated by Dr Sheila Polakoff, assisted by Mrs Patricia Mulliss and Mrs Jean Miller, Heptatis Epidemiology Unit Central Public Health Laboratory.

We are indebted to the many physicians who helped us obtain clinical and epidemiological data.

\section{References}

1 Polakoff S, Tillett HE. Acute viral hepatitis B: laboratory reports 1975-9. Br Med f 1982;284:1881 2.

2 Pattison CP, Maynard JE, Berquist KR, Webster HM. Epidemiology of hepatitis B in hospital personnel. Am f Epidemiol 1975;101:59-64.

Report of a collaborative study. Acute hepatitis B associated with gynaecological surgery. Lance 1980;i:1-6.

4 Department of Health and Social Security. Hospital In-Patient Enquiry 1981. HMSO, London: 1981 (Series MB4 No 17-18).

5 Department of Health and Social Security. Hepatitis B and NHS Staff. London: DHSS, 1981 (CMO $(81) 11$.) 6 Stewart JS, Farrow LJ, Clifford, RE, et al. A three-year survey of viral hepatitis in West London. $f$

7 Polakoff S, Tillett HE. Routine laboratory reports of patients with acute hepatitis $B$ as indicators of incidence of the disease. Foumal of Infection 1984;8:44-8.

(Accepted 27 March 1986) , . . . 离 .

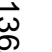

\section{.}

\section{MATERIA NON MEDICA}

\section{Potion Puense}

Economising is essential, earning a little money is better. In a small and (very) rural hospital on the Zairean/Ugandan border somewhere between the Rwenzori mountains and Lake Albert-or Lac Mobutu, as we say heretransport weighs heavily on the budget. The running costs of the hospital have to be paid, directly or indirectly, by the population. Therefore, while maintaining the quality of treatment, we must keep our medical activities as simple and cheap as possible.

The rainy season helps. It provides clean water, straight from the roof, much better than the heavily jaundiced fluid that comes from the spring. The ideal water for intravenous fluids. Just add the right amount of domestic salt (non-iodised of course), filter, and sterilise it. Charcoal for the autoclave is cheap. Anybody working under similar conditions knows the trick.

We also make a few home brewed syrups and suspensions, particularly for children. This is much cheaper than buying it from the nearest, but still far away, pharmacy. After a little experimenting I found a nice formula for cough mixture, successful therapeutically as well as financially: cheap for the patients while not unprofitable for the hospital. After all, staff salaries have to come from somewhere. The recipe, carefully kept secret until now, is as follows:

Wild honey (found in the forest and for sale at the village market) 1 litre Strong tea brewed from eucalyptus leaves (available everywhere) 2 litres Ginger powder

3 big spoonfuls

This makes 3 litres of our basic cough mixture. Crushed ephedrine tablets or phenergan can be added if desired. Because honey is the main ingredient I called it Potion Puense. No doubt many readers will recognise Puense as the official Latin adjective derived from the name of the most famous honey addict in English literature. ${ }^{1}$

The cost price comes to $0.21 \mathrm{p} / 5 \mathrm{ml}$. We think it is reasonable to sell it for $3.75 p$, which is less than what people used to pay for cough mixture from the pharmacy. Potion Puense is rapidly becoming popular and goes down very well, even in those children who have not yet read Winnie ille Pu.

Would anybody in the NHS be interested in bypassing the pharmaceutical industry? We could of course send you a regular supply of eucalyptus leaves. Our prices are very reasonable.-CHRISTINA M DE WIND, Haut Zaire.

1 Staples B. Domus Anguli Puensis: a Latin version of A A Milne's The House at Pooh Corner. London: Methuen Children's Books, 1980. 